\title{
Numerical Solutions of Odd Order Linear and Nonlinear Initial Value Problems Using a Shifted Jacobi Spectral Approximations
}

\author{
A. H. Bhrawy ${ }^{1,2}$ and M. A. Alghamdi ${ }^{1}$ \\ ${ }^{1}$ Department of Mathematics, Faculty of Science, King Abdulaziz University, Jeddah 21589, Saudi Arabia \\ ${ }^{2}$ Department of Mathematics, Faculty of Science, Beni-Suef University, Beni-Suef 62511, Egypt
}

Correspondence should be addressed to A. H. Bhrawy, alibhrawy@yahoo.co.uk

Received 25 May 2012; Accepted 26 June 2012

Academic Editor: D. Anderson

Copyright (c) 2012 A. H. Bhrawy and M. A. Alghamdi. This is an open access article distributed under the Creative Commons Attribution License, which permits unrestricted use, distribution, and reproduction in any medium, provided the original work is properly cited.

A shifted Jacobi Galerkin method is introduced to get a direct solution technique for solving the third- and fifth-order differential equations with constant coefficients subject to initial conditions. The key to the efficiency of these algorithms is to construct appropriate base functions, which lead to systems with specially structured matrices that can be efficiently inverted. A quadrature Galerkin method is introduced for the numerical solution of these problems with variable coefficients. A new shifted Jacobi collocation method based on basis functions satisfying the initial conditions is presented for solving nonlinear initial value problems. Through several numerical examples, we evaluate the accuracy and performance of the proposed algorithms. The algorithms are easy to implement and yield very accurate results.

\section{Introduction}

The spectral methods are preferable in numerical solutions of ordinary and partial differential equations due to their high-order accuracy whenever they work [1-3]. Standard spectral and collocation methods have been extensively investigated for solving second- and fourth-order differential equations. In a sequence of papers [4-11], the authors have constructed efficient spectral-Galerkin algorithms for second-, fourth-, and $2 n$ th-order differential equations subject to various boundary conditions.

The problem of approximating solutions of differential equations by Galerkin approximations involves the projection onto the span of some appropriate set of basis functions. The member of the basis may satisfy automatically the auxiliary conditions imposed on the problem, such as initial, boundary, or more general conditions. Alternatively, these conditions 
may be imposed as constraints on the expansions coefficients, as in the Lanczos tau-method [12-14].

It is of fundamental importance to know that the choice of the basis functions is responsible for the superior approximation properties of spectral methods when compared with the finite difference and finite element methods. The choice of different basis functions lead to different spectral approximations; for instance, trigonometric polynomials for periodic problems, Chebyshev, Legendre, ultraspherical, and Jacobi polynomials for nonperiodic problems, Laguerre polynomials for problems on half line, and Hermite polynomials for problems on the whole line.

The main aim of this paper is the design of appropriate shifted Jacobi basis (with parameters $\alpha$ and $\beta$ ) that are well suited for the approximations of the third- and fifth-order differential equations subject to initial conditions. In general, the use of Jacobi polynomials $\left(P_{n}^{(\alpha, \beta)}\right.$ with $\alpha, \beta \in(-1, \infty)$ and $n$ is the polynomial degree) has the advantage of obtaining the solutions of differential equations in terms of the Jacobi indexes $\alpha$ and $\beta$ (see for instance, [15-19]).

This paper is concerned with the systematic development of spectral basis functions for the efficient solution of some odd-order differential equations. Starting from Jacobi polynomials $P_{n}^{(\alpha, \beta)}(x)$. Galerkin approximations to these problems are built. We derived some interesting results, such as useful relationships between the representation of a polynomial function in a given basis and those for its derivative in the same basis, or formulas to compute discrete operator coefficients in closed form. In this paper, we present a direct solvers based on the shifted Jacobi Galerkin (SJG) method for solving the third- and fifth-order differential equations, the basis functions are constructed to satisfy the given initial conditions, and each of these basis functions have been written as a compact combinations of shifted Jacobi polynomials.

For the third- and fifth-order differential equations with variable coefficients, we introduce the pseudospectral shifted Jacobi Galerkin (P-SJG) method. This method is basically formulated in the shifted Jacobi Galerkin spectral form with general indexes $\alpha, \beta>-1$, but the variable coefficients terms and the right hand side being treated by the shifted Jacobi collocation method with the same indexes $\alpha, \beta>-1$ so that the schemes can be implemented at shifted Jacobi-Gauss points efficiently.

The last aim of this paper is to propose a suitable way to approximate the nonlinear third- and fifth-order differential equations by convenient spectral collocation method-based on shifted Jacobi basis functions (the member of the basis may satisfy automatically the auxiliary initial conditions imposed on the problem) such that it can be implemented efficiently at shifted Jacobi-Gauss points on the interval $(0, L)$. We propose a new spectral shifted Jacobi collocation (SJC) method to find the solution $u_{N}(x)$. The nonlinear ODE is collocated at the $(N+1)$ points. For suitable collocation points, we use the $(N+1)$ nodes of the shifted Jacobi-Gauss interpolation on $(0, L)$. These equations generate $(N+1)$ nonlinear algebraic equations which can be solved using Newton's iterative method. Finally, the accuracy of the proposed methods is demonstrated by test problems. Numerical results are presented in which the usual exponential convergence behaviour of spectral approximations is exhibited.

The remainder of this paper is organized as follows. Sections 2 and 3 are devoted to the theoretical derivation of the SJG and P-SJG methods for third-order differential equations with constant and variable coefficients subject to homogeneous and nonhomogeneous initial conditions. In Section 4, we apply the SJC method-based on basis functions for solving 
nonlinear third-order differential equations. Section 5 gives the corresponding results for those obtained in Sections 2, 3, and 4, but for the fifth-order differential equations. In Section 6, we present some numerical results exhibiting the accuracy and efficiency of our numerical algorithms.

\section{SJG Method for Third-Order Differential Equations with Constant Coefficients}

Let $w^{(\alpha, \beta)}(x)=(1-x)^{\alpha}(1+x)^{\beta}$, then we define the weighted space $L_{w^{(\alpha, \beta)}}^{2}(-1,1)$ as usual, equipped with the following inner product and norm,

$$
(u, v)_{w^{(\alpha, \beta)}}=\int_{-1}^{1} u(x) v(x) w^{(\alpha, \beta)}(x) d x, \quad\|v\|_{w^{(\alpha, \beta)}}=(v, v)_{w^{(\alpha, \beta)}}^{1 / 2}
$$

The set of Jacobi polynomials forms a complete $L_{w^{\alpha, \beta}}^{2}(-1,1)$-orthogonal system, and

$$
\left\|P_{k}^{(\alpha, \beta)}\right\|_{w^{(\alpha, \beta)}}^{2}=h_{k}^{(\alpha, \beta)}=\frac{2^{\alpha+\beta+1} \Gamma(k+\alpha+1) \Gamma(k+\beta+1)}{(2 k+\alpha+\beta+1) \Gamma(k+1) \Gamma(k+\alpha+\beta+1)} .
$$

If we define the shifted Jacobi polynomial of degree $k$ by $P_{L, k}^{(\alpha, \beta)}(x)=P_{k}^{(\alpha, \beta)}(2 x / L-1)$, $L>0$, and in virtue of properties of Jacobi polynomials $[14,19]$, then it can be easily shown that

$$
\begin{gathered}
P_{L, k}^{(\alpha, \beta)}(0)=(-1)^{k} \frac{\Gamma(k+\beta+1)}{\Gamma(\beta+1) k !} \\
D^{q} P_{L, k}^{(\alpha, \beta)}(0)=\frac{(-1)^{k-q} \Gamma(k+\beta+1)(k+\alpha+\beta+1)_{q}}{L^{q} \Gamma(k-q+1) \Gamma(q+\beta+1)} .
\end{gathered}
$$

Next, let $w_{L}^{(\alpha, \beta)}(x)=(L-x)^{\alpha} x^{\beta}$, then we define the weighted space $L_{w_{L}^{(\alpha, \beta)}}^{2}(0, L)$ in the usual way, with the following inner product and norm,

$$
(u, v)_{w_{L}^{(\alpha, \beta)}}=\int_{0}^{L} u(x) v(x) w_{L}^{(\alpha, \beta)}(x) d x, \quad\|v\|_{w_{L}^{(\alpha, \beta)}}=(v, v)_{w_{L}^{(\alpha, \beta)}}^{1 / 2}
$$

The set of shifted Jacobi polynomials forms a complete $L_{w_{L}^{(\alpha, \beta)}}^{2}(0, L)$-orthogonal system. Moreover, and due to (2.2), we have

$$
\left\|P_{L, k}^{(\alpha, \beta)}\right\|_{w_{L}^{(\alpha, \beta)}}^{2}=\left(\frac{L}{2}\right)^{\alpha+\beta+1} h_{k}^{(\alpha, \beta)}=h_{L, k}^{(\alpha, \beta)} .
$$


The $q$ th derivative of shifted Jacobi polynomial can be written in terms of the shifted Jacobi polynomials themselves as

$$
D^{q} P_{L, k}^{(\alpha, \beta)}(x)=\sum_{i=0}^{k-q} C_{q}(k, i, \alpha, \beta) P_{L, i}^{(\alpha, \beta)}(x),
$$

where

$$
\begin{aligned}
C_{q}(k, i, \alpha, \beta)= & \frac{(k+\lambda)_{q}(k+\lambda+q)_{i}(i+\alpha+q+1)_{k-i-q} \Gamma(i+\lambda)}{L^{q}(k-i-q) ! \Gamma(2 i+\lambda)} \\
& \times{ }_{3} F_{2}\left(\begin{array}{lll}
-k+i+q, & k+i+\lambda+q, i+\alpha+1 & \\
i+\alpha+q+1, & 2 i+\lambda+1 & ; 1
\end{array}\right),
\end{aligned}
$$

for the proof, see $[20,21]$ and for the general definition of a generalized hypergeometric series and special ${ }_{3} F_{2}$, (see [22, pages $\left.41,103-104\right]$, resp.). equation:

We are interested in using the SJG method to solve the third-order differential

$$
u^{\prime \prime \prime}+\gamma_{1} u^{\prime \prime}+\gamma_{2} u^{\prime}+\gamma_{3} u=f(x), \quad \text { in } I=(0, L),
$$

subject to

$$
u(0)=u^{\prime}(0)=u^{\prime \prime}(0)=0,
$$

where $\gamma_{1}, \gamma_{2}$, and $\gamma_{3}$ are constants, and $f(x)$ is a given source function. Let us first introduce some basic notation that will be used in the upcoming sections. We set

$$
\begin{aligned}
& S_{N}(0, L)=\operatorname{span}\left\{P_{L, 0}^{(\alpha, \beta)}(x), P_{L, 1}^{(\alpha, \beta)}(x), \ldots, P_{L, N}^{(\alpha, \beta)}(x)\right\}, \\
& W_{N}=\left\{v_{N} \in S_{N}(0, L): u(0)=u^{\prime}(0)=u^{\prime \prime}(0)=0\right\} .
\end{aligned}
$$

Then the shifted Jacobi-Galerkin approximation to (2.9) is, to find $u_{N} \in W_{N}$ such that

$$
\begin{aligned}
& \left(u_{N}^{\prime \prime \prime}, v_{N}\right)_{w_{L}^{(\alpha, \beta)}}+\gamma_{1}\left(u_{N}^{\prime \prime}, v_{N}\right)_{w_{L}^{(\alpha, \beta)}}+\gamma_{2}\left(u_{N^{\prime}}^{\prime}, v_{N}\right)_{w_{L}^{(\alpha, \beta)}}+\gamma_{3}\left(u_{N}, v_{N}\right)_{w_{L}^{(\alpha, \beta)}} \\
& =\left(f, v_{N}\right)_{w_{L}^{(\alpha, \beta)},}, \quad \forall v_{N} \in W_{N}
\end{aligned}
$$

where $w_{L}^{(\alpha, \beta)}(x)=(L-x)^{\alpha} x^{\beta}$ and $(u, v)_{w_{L}^{(\alpha, \beta)}}=\int_{I} u v w_{L}^{\alpha, \beta} d x$ is the inner product in the weighted space $L_{w_{L}^{(\alpha, \beta)}}^{2}(I)$. The norm in $L_{w_{L}^{(\alpha, \beta)}}^{2}(I)$ will be denoted by $\|\cdot\|_{w_{L}^{(\alpha, \beta)}}$. 
We choose compact combinations of shifted Jacobi polynomials as basis functions aiming to minimize the bandwidth and the condition number of the coefficient matrix corresponding to (2.9). We choose the basis functions of expansion $\phi_{k}(x)$ to be of the form:

$$
\phi_{k}(x)=\xi_{k}\left[P_{L, k}^{(\alpha, \beta)}(x)+\epsilon_{k} P_{L, k+1}^{(\alpha, \beta)}(x)+\varepsilon_{k} P_{L, k+2}^{(\alpha, \beta)}(x)+\zeta_{k} P_{L, k+3}^{(\alpha, \beta)}(x)\right]
$$

where $\xi_{k}=k ! \Gamma(\alpha+1) / \Gamma(k+\alpha+1), \epsilon_{k}, \varepsilon_{k}$, and $\zeta_{k}$ are the unique constants such that $\phi_{k}(x) \in W_{N}$, for all $k=0,1, \ldots, N-3$. From the initial conditions; $\phi_{k}(0)=\phi_{k}^{\prime}(0)=\phi_{k}^{\prime \prime}(0)=0$ and making use of (2.3) and (2.4), we have the following system:

$$
\begin{gathered}
-\epsilon_{k} \frac{(k+\beta+1)}{(k+1)}+\epsilon_{k} \frac{(k+\beta+1)_{2}}{(k+1)_{2}}-\zeta_{k} \frac{(k+\beta+1)_{3}}{(k+1)_{3}}=-1, \\
\epsilon_{k} \frac{(k+\lambda+1)(k+\beta+1)}{k}-\varepsilon_{k} \frac{(k+\lambda+2)(k+\beta+1)_{2}}{k(k+1)} \\
+\zeta_{k} \frac{(k+\lambda+3)(k+\beta+1)_{3}}{k(k+1)_{2}}=(k+\lambda), \\
-\epsilon_{k} \frac{(k+\lambda+1)_{2}(k+\beta+1)}{(k-1)}+\varepsilon_{k} \frac{(k+\lambda+2)_{2}(k+\beta+1)_{2}}{k(k-1)} \\
-\zeta_{k} \frac{(k+\lambda+3)_{2}(k+\beta+1)_{3}}{(k+1) k(k-1)}=-(k+\lambda)_{2} .
\end{gathered}
$$

Hence $\epsilon_{k}, \varepsilon_{k}$, and $\zeta_{k}$ can be uniquely determined to give

$$
\begin{gathered}
\epsilon_{k}=\frac{3(k+1)(2 k+\lambda+2)}{(k+\beta+1)(2 k+\jmath+4)}, \\
\varepsilon_{k}=\frac{3(k+1)_{2}(2 k+\lambda+1)}{(k+\beta+1)_{2}(2 k+\lambda+5)}, \\
\zeta_{k}=\frac{(k+1)_{3}(2 k+\lambda+1)_{2}}{(k+\beta+1)_{3}(2 k+\lambda+4)_{2}} .
\end{gathered}
$$

It is clear that the basis functions $\phi_{k}(x) \in W_{k+3}, k=0,1,2, \ldots, N-3$, are linearly independent. Therefore, by dimension argument and for $N \geq 3$, we have

$$
W_{N}=\operatorname{span}\left\{\phi_{k}(x): k=0,1,2, \ldots, N-3\right\} .
$$

Now, it is clear that the variational formulation of (2.12) is equivalent to

$$
\begin{aligned}
& \left(u_{N^{\prime}}^{\prime \prime \prime} \phi_{k}(x)\right)_{w_{L}^{(\alpha, \beta)}}+\gamma_{1}\left(u_{N^{\prime}}^{\prime \prime} \phi_{k}(x)\right)_{w_{L}^{(\alpha, \beta)}}+\gamma_{2}\left(u_{N}^{\prime}, \phi_{k}(x)\right)_{w_{L}^{(\alpha, \beta)}}+\gamma_{3}\left(u_{N}, \phi_{k}(x)\right)_{w_{L}^{(\alpha, \beta)}} \\
& =\left(f, \phi_{k}(x)\right)_{w_{T}^{(\alpha, \beta)},} \quad k=0,1, \ldots, N-3 .
\end{aligned}
$$


Let us denote

$$
\begin{gathered}
f_{k}=\left(f, \phi_{k}(x)\right)_{w_{L}^{(\alpha, \beta)}, \quad \mathbf{f}=\left(f_{0}, f_{1}, \ldots, f_{N-3}\right)^{T},} \\
u_{N}(x)=\sum_{n=0}^{N-3} a_{n} \phi_{n}(x), \quad \mathbf{a}=\left(a_{0}, a_{1}, \ldots, a_{N-3}\right)^{T}, \\
A=\left(a_{k j}\right), \quad B=\left(b_{k j}\right), \quad C=\left(c_{k j}\right), \quad D=\left(d_{k j}\right), \quad 0 \leq k, j \leq N-3 .
\end{gathered}
$$

Then, equation (2.19) is equivalent to the following matrix equation:

$$
\left(A+\gamma_{1} B+\gamma_{2} C+\gamma_{3} D\right) \mathbf{a}=\mathbf{f}
$$

where the nonzero elements of the matrices $A, B, C$, and $D$ are given explicitly in the following theorem.

Theorem 2.1. If one takes $\phi_{k}(x)$ as defined in (2.13), and if we denote $a_{k j}=\left(\phi_{j}^{\prime \prime \prime}(x), \phi_{k}(x)\right)_{w_{L}^{(\alpha, \beta)}}$,

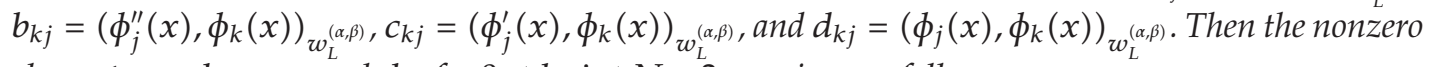
elements $a_{k j}, b_{k j}, c_{k j}$, and $d_{k j}$ for $0 \leq k, j \leq N-3$ are given as follows:

$$
\begin{aligned}
& a_{k k}=\frac{L^{\alpha+\beta-2}(2 k+\lambda+1)_{3}(2 k+\lambda+1)_{2} \Gamma(k+4)(\Gamma(\alpha+1))^{2} \Gamma(k+\beta+1)}{(k+\beta+1)_{3} \Gamma(k+\alpha+1) \Gamma(k+\lambda+3)}, \\
& a_{k j}=\xi_{k} \xi_{j}\left[O_{3}(j, k, \alpha, \beta) h_{L, k}^{(\alpha, \beta)}+O_{3}(j, k+1, \alpha, \beta) \epsilon_{k} h_{L, k+1}^{(\alpha, \beta)}+O_{3}(j, k+2, \alpha, \beta) \varepsilon_{k} h_{L, k+2}^{(\alpha, \beta)}\right. \\
& \left.+O_{3}(j, k+3, \alpha, \beta) \zeta_{k} h_{L, k+3}^{(\alpha, \beta)}\right], \quad j=k+n, n \geq 1, \\
& b_{k+1, k}=\xi_{k} \xi_{k+1} \zeta_{k} C_{2}(k+3, k+1, \alpha, \beta) h_{L, k+1}^{(\alpha, \beta)}, \\
& b_{k k}=\xi_{k}^{2}\left[\left(\varepsilon_{k} C_{2}(k+2, k, \alpha, \beta)+\zeta_{k} C_{2}(k+3, k, \alpha, \beta)\right) h_{L, k}^{(\alpha, \beta)}\right. \\
& \left.+\zeta_{k} \epsilon_{k} C_{2}(k+3, k+1, \alpha, \beta) h_{L, k+1}^{(\alpha, \beta)}\right], \\
& \left.\quad+O_{2}(j, k+3, \alpha, \beta) \zeta_{k} h_{L, k+3}^{(\alpha, \beta)}\right], \quad j=k+n, n \geq 1,
\end{aligned}
$$




$$
\begin{gathered}
c_{k+2, k}=\xi_{k} \xi_{k+2} \zeta_{k} C_{1}(k+3, k+2, \alpha, \beta) h_{L, k+2,}^{(\alpha, \beta)} \\
c_{k+1, k}=\xi_{k} \xi_{k+1}\left[\left(\varepsilon_{k} C_{1}(k+2, k+1, \alpha, \beta)+\zeta_{k} C_{1}(k+3, k+1, \alpha, \beta)\right) h_{L, k+1}^{(\alpha, \beta)}\right. \\
\left.\quad+\zeta_{k} \epsilon_{k+1} C_{1}(k+3, k+2, \alpha, \beta) h_{L, k+2}^{(\alpha, \beta)}\right] \\
c_{k k}=\xi_{k}^{2}\left[\left(\epsilon_{k} C_{1}(k+1, k, \alpha, \beta)+\varepsilon_{k} C_{1}(k+2, k, \alpha, \beta)+\zeta_{k} C_{1}(k+3, k, \alpha, \beta)\right) h_{L, k}^{(\alpha, \beta)}\right. \\
+\epsilon_{k}\left(\varepsilon_{k} C_{1}(k+2, k+1, \alpha, \beta)+\zeta_{k} C_{1}(k+3, k+1, \alpha, \beta)\right) h_{L, k+1}^{(\alpha, \beta)} \\
\left.+\zeta_{k} \varepsilon_{k} C_{1}(k+3, k+2, \alpha, \beta) h_{L, k+2}^{(\alpha, \beta)}\right], \\
c_{k j}=\xi_{k} \xi_{j}\left[O_{1}(j, k, \alpha, \beta) h_{L, k}^{(\alpha, \beta)}+O_{1}(j, k+1, \alpha, \beta) \epsilon_{k} h_{L, k+1}^{(\alpha, \beta)}+O_{1}(j, k+2, \alpha, \beta) \varepsilon_{k} h_{L, k+2}^{(\alpha, \beta)}\right. \\
\left.+O_{1}(j, k+3, \alpha, \beta) \zeta_{k} h_{L, k+3}^{(\alpha, \beta)}\right], \quad j=k+n, n \geq 1, \\
d_{k k}=\xi_{k}^{2}\left[h_{L, k}^{(\alpha, \beta)}+\epsilon_{k}^{2} h_{L, k+1}^{(\alpha, \beta)}+\varepsilon_{k}^{2} h_{L, k+2}^{(\alpha, \beta)}+\zeta_{k}^{2} h_{L, k+3}^{(\alpha, \beta)}\right], \\
d_{k+1, k}=d_{k, k+1}=\xi_{k} \xi_{k+1}\left[\epsilon_{k} h_{L, k+1}^{(\alpha, \beta)}+\epsilon_{k+1} \varepsilon_{k} h_{L, k+2}^{(\alpha, \beta)}+\varepsilon_{k+1} \zeta_{k} h_{L, k+3}^{(\alpha, \beta)}\right] \\
d_{k+2, k}=d_{k, k+2}=\xi_{k} \xi_{k+2}\left[\varepsilon_{k} h_{L, k+2}^{(\alpha, \beta)}+\epsilon_{k+2} \zeta_{k} h_{L, k+3}^{(\alpha, \beta)}\right], \quad d_{k+3, k}=d_{k, k+3}=\xi_{k} \xi k+3 \zeta_{k} h_{L, k+3}^{(\alpha, \beta)}
\end{gathered}
$$

where

$$
\begin{aligned}
O_{i}(j, k, \alpha, \beta)= & C_{i}(j, k, \alpha, \beta)+\epsilon_{j} C_{i}(j+1, k, \alpha, \beta)+\varepsilon_{j} C_{i}(j+2, k, \alpha, \beta) \\
& +\zeta_{j} C_{i}(j+3, k, \alpha, \beta) .
\end{aligned}
$$

Proof. The basis functions $\phi_{k}(x)$ are chosen such that $\phi_{k}(x) \in W_{N}$ for $k=0,1, \ldots, N-3$. On the other hand, it is clear that $\left\{\phi_{k}(x)\right\}$ are linearly independent and the dimension of $W_{N}$ is equal to $(N-2)$. The nonzero elements $\left(a_{k j}\right)$ for $0 \leqslant k, j \leqslant N-3$ can be obtained by direct computations using the properties of shifted Jacobi polynomials. It can be easily proved that the diagonal elements of the matrix A take the form:

$$
a_{k k}=\xi_{k}^{2} \zeta_{k} C_{3}(k+3, k, \alpha, \beta) h_{L, k}^{(\alpha, \beta)}
$$

It can be easily shown, that all other formulae can be obtained by direct computations using the properties of shifted Jacobi polynomials.

All the formulae can be obtained by direct computations using the properties of shifted Jacobi polynomials. In particular, the special cases for shifted Chebyshev basis of the first and second kinds may be obtained directly by taking $\alpha=\beta=-1 / 2$ and $\alpha=\beta=1 / 2$, respectively, and for shifted Legendre basis by taking $\alpha=\beta=0$. These are given as corollaries to the previous theorem as follows. 
Corollary 2.2. If $\alpha=\beta=0$, then the nonzero elements $\left(a_{k j}\right),\left(b_{k j}\right),\left(c_{k j}\right),\left(d_{k j}\right)$ for $0 \leq k, j \leq N-3$ are given as follows:

$$
\begin{aligned}
& a_{k k}=\frac{8(k+1)(2 k+3)^{2}}{L^{2}(k+3)}, \\
& a_{k j}=\frac{8(2 j+3)(2 k+3)}{L^{2}(j+3)(k+3)} \\
& \times\left[k^{4}+8 k^{3}+\left(17-8 j-2 j^{2}\right) k^{2}+4\left(1-8 j-2 j^{2}\right) k+\left(j^{4}+8 j^{3}+17 j^{2}+16\right)\right], \\
& j=k+n, n \geq 1, \\
& b_{k+1, k}=\frac{4(k+1)(2 k+3)}{L(k+3)}, \quad b_{k k}=\frac{48(k+1)(k+2)(2 k+3)}{L(k+3)(2 k+5)}, \\
& b_{k, k+1}=\frac{4(2 k+3)\left(17 k^{2}+85 k+104\right)}{L(k+3)(k+4)}, \\
& b_{k j}=\frac{16(2 j+3)(j-k)(j+k+4)(2 k+3)}{L(j+3)(k+3)}, \quad j=k+n, n \geq 2, \\
& c_{k+2, k}=\frac{2(k+1)(2 k+3)}{(k+3)(2 k+5)}, \quad c_{k+1, k}=\frac{12(k+1)(2 k+5)}{(k+3)(2 k+7)}, \\
& c_{k k}=\frac{8(2 k+3)^{2}}{(k+3)^{2}}, \quad c_{k, k+1}=\frac{4(2 k+5)\left(13 k^{2}+65 k+72\right)}{(k+3)(k+4)(2 k+7)}, \\
& c_{k, k+2}=\frac{2(2 k+3)\left(31 k^{2}+186 k+275\right)}{(k+3)(k+5)(2 k+5)}, \\
& c_{k j}=\frac{16(2 j+3)(2 k+3)}{(j+3)(k+3)}, \quad j=k+n, n \geq 3, \\
& d_{k k}=\frac{40 L(k+1)(k+2)(2 k+3)}{(k+3)(2 k+1)(2 k+5)(2 k+7)}, \quad d_{k, k+1}=d_{k+1, k}=\frac{15 L(k+2)}{(k+4)(2 k+7)}, \\
& d_{k, k+2}=d_{k+2, k}=\frac{12 L(k+1)}{(2 k+5)(2 k+9)}, \quad d_{k, k+3}=d_{k+3, k}=\frac{L(k+1)(2 k+3)}{(k+3)(2 k+5)(2 k+7)} .
\end{aligned}
$$

Corollary 2.3. If $\alpha=\beta=1 / 2$, then the nonzero elements $\left(a_{k j}\right),\left(b_{k j}\right),\left(c_{k j}\right)$, and $\left(d_{k j}\right)$ for $0 \leq k, j \leq$ $N-3$ are given as follows:

$$
a_{k k}=\frac{8(k+2)^{2}(2 k+3) \pi}{L(k+4)(2 k+7)}
$$




$$
\begin{aligned}
& a_{k j}=\frac{4(2 j+3)(2 j+5)(k+2) \pi}{L(j+1)(j+3)(j+4)(2 k+7)} \\
& \times\left[k^{4}+10 k^{3}+(29-2 j(j+5)) k^{2}-10(j(j+5)-2) k+j(j+5)(j(j+5)-2)+12\right], \\
& j=k+n, n \geq 1, \\
& b_{k+1, k}=\frac{2(k+2)(2 k+3) \pi}{(k+4)(2 k+7)}, \quad b_{k k}=\frac{12(k+2)(2 k+3) \pi}{(k+4)(2 k+7)}, \\
& b_{k, k+1}=\frac{2(k+2)(k+3)(34 k+115) \pi}{(k+4)(k+5)(2 k+7)}, \\
& b_{k j}=\frac{4(2 j+3)(2 j+5)(k+2)\left(2 j^{2}+10 j-2 k^{2}-10 k-3\right) \pi}{(j+1)(j+3)(j+4)(2 k+7)}, \quad j=k+n, n \geq 2, \\
& c_{k+2, k}=\frac{L(k+2)(2 k+3) \pi}{2(k+3)(k+4)(2 k+7)}, \quad c_{k+1, k}=\frac{3 L(2 k+3)\left(2 k^{2}+13 k+22\right) \pi}{2(k+3)(k+4)^{2}(2 k+7)}, \\
& c_{k k}=\frac{L\left(64 k^{4}+700 k^{3}+2860 k^{2}+5187 k+3564\right) \pi}{2(k+3)^{2}(k+4)(2 k+7)^{2}}, \\
& c_{k, k+1}=\frac{L\left(52 k^{4}+560 k^{3}+2249 k^{2}+3985 k+2640\right) \pi}{2(k+2)(k+3)(k+4)(k+5)(2 k+7)}, \\
& c_{k, k+2}=\frac{L(k+2)\left(62 k^{3}+679 k^{2}+2451 k+2934\right) \pi}{2(k+3)^{2}(k+5)(k+6)(2 k+7)}, \\
& c_{k j}=\frac{8 L(2 j+5)(2 j+7)(k+2) \pi}{(j+2)(j+4)(j+5)(2 k+7)}, \quad j=k+n, n \geq 3, \\
& d_{k k}=\frac{L^{2}\left(20 k^{6}+300 k^{5}+1841 k^{4}+5910 k^{3}+10478 k^{2}+9765 k+3861\right) \pi}{2(k+1)^{2}(k+3)^{2}(k+4)^{2}(2 k+7)^{2}}, \\
& d_{k, k+1}=d_{k+1, k}=\frac{3 L^{2}\left(20 k^{4}+240 k^{3}+1087 k^{2}+2202 k+1716\right) \pi}{8(k+2)(k+3)(k+4)^{2}(2 k+7)(2 k+9)}, \\
& d_{k, k+2}=d_{k+2, k}=\frac{3 L^{2}(2 k+3)\left(k^{2}+7 k+13\right) \pi}{4(k+3)^{2}(k+4)(k+5)(2 k+7)}, \\
& d_{k, k+3}=d_{k+3, k}=\frac{L^{2}(k+2)(2 k+3) \pi}{8(k+3)(k+4)^{2}(2 k+7)} .
\end{aligned}
$$

Corollary 2.4. If $\alpha=\beta=-1 / 2$, then the nonzero elements $\left(a_{k j}\right),\left(b_{k j}\right),\left(c_{k j}\right)$, and $\left(d_{k j}\right)$ for $0 \leq k$, $j \leq N-3$ are given as follows:

$$
a_{k k}=\frac{32(k+1)^{2}(k+3)(2 k+1) \pi}{L^{3}(2 k+5)}
$$




$$
\begin{aligned}
& a_{k j}=\frac{16(2 j+1)(2 j+3)(k+1) \pi}{L^{3}(j+2)(2 k+5)} \\
& \times\left[k^{4}+6 k^{3}+(7-2 j(3+j)) k^{2}-6(1+j(3+j)) k+\left(2+3 j+j^{2}\right)^{2}\right], \\
& j:=k+n, n \geq 1, \\
& b_{k+1, k}=\frac{8(k+1)(k+3)(2 k+1) \pi}{L^{2}(2 k+5)}, \quad b_{k k}=\frac{24(k+1)(2 k+1)\left(2 k^{2}+8 k+7\right) \pi}{L^{2}(k+2)(2 k+5)}, \\
& b_{k, k+1}=\frac{8(k+1)\left(34 k^{3}+225 k^{2}+482 k+333\right) \pi}{L^{2}(k+3)(2 k+5)}, \\
& b_{k j}=\frac{16(2 j+1)(2 j+3)(k+1)\left(2 j^{2}+6 j-2 k^{2}-6 k+3\right) \pi}{L^{2}(j+2)(2 k+5)}, \quad j=k+n, n \geq 2 \text {, } \\
& c_{k+2, k}=\frac{2(k+1)(k+3)(2 k+1) \pi}{L(k+2)(2 k+5)}, \quad c_{k+1, k}=\frac{6(2 k+1)(2 k+3) \pi}{L(2 k+5)}, \\
& c_{k k}=\frac{2(k+1)\left(64 k^{3}+324 k^{2}+476 k+189\right) \pi}{L(k+2)(2 k+5)^{2}}, \\
& c_{k, k+1}=\frac{2(k+1)(26 k+45) \pi}{L(k+3)}, \quad c_{k, k+2}=\frac{2(k+1)\left(62 k^{2}+375 k+556\right) \pi}{L(k+4)(2 k+5)}, \\
& c_{k j}=\frac{32(2 j+1)(2 j+3)(k+1) \pi}{L(j+2)(2 k+5)}, \quad j=k+n, n \geq 3, \\
& d_{k k}=\frac{\left(40 k^{4}+240 k^{3}+526 k^{2}+498 k+181\right) \pi}{(k+2)^{2}(2 k+5)^{2}} \\
& d_{k, k+1}=d_{k+1, k}=\frac{3\left(20 k^{4}+160 k^{3}+451 k^{2}+524 k+198\right) \pi}{2(k+2)(k+3)(2 k+5)(2 k+7)} \\
& d_{k, k+2}=d_{k+2, k}=\frac{3(2 k+1)\left(2 k^{2}+10 k+11\right) \pi}{2(k+2)(k+4)(2 k+5)}, \\
& d_{k, k+3}=d_{k+3, k}=\frac{(k+1)(2 k+1) \pi}{2(k+2)(2 k+5)} \text {. }
\end{aligned}
$$

In the following, we can always modify the right-hand side to take care of the nonhomogeneous initial conditions. Let us consider for instance the one-dimensional third-order differential equation (2.9) subject to the nonhomogeneous initial conditions:

$$
u(0)=a_{+}, \quad u^{\prime}(0)=a_{-}, \quad u^{\prime \prime}(0)=\tilde{a}_{+} .
$$

We proceed as follows. 


\section{Set}

$$
V(x)=u(x)+b_{0}+b_{1} x+b_{2} x^{2}
$$

where

$$
b_{0}=-a_{+}, \quad b_{1}=-a_{-,} \quad b_{2}=\frac{-\tilde{a}_{+}}{2} .
$$

The transformation (2.29) turns the nonhomogeneous initial conditions (2.28) into the homogeneous initial conditions:

$$
V(0)=V^{\prime}(0)=V^{\prime \prime}(0)=0
$$

Hence, it suffices to solve the following modified one-dimensional third-order differential equation:

$$
V^{\prime \prime \prime}+\gamma_{1} V^{\prime \prime}+\gamma_{2} V^{\prime}+\gamma_{3} V=f^{*}(x) \text { in } I=(0, L),
$$

subject to the homogeneous initial conditions (2.31), where $V(x)$ is given by (2.29), and

$$
f^{*}(x)=f(x)+\left(\gamma_{3} b_{0}+\gamma_{2} b_{1}+2 \gamma_{1} b_{2}\right)+\left(\gamma_{3} b_{1}+2 \gamma_{2} b_{2}\right) x+\gamma_{3} b_{2} x^{2}
$$

\section{P-SJG Method for Third-Order Differential Equation with Variable Coefficients}

In this section, we use the pseudospectral-shifted Jacobi Galerkin method to numerically solve the following third-order differential equation with variable coefficients:

$$
\begin{gathered}
u^{\prime \prime \prime}+\gamma_{1}(x) u^{\prime \prime}+\gamma_{2}(x) u^{\prime}+\gamma_{3}(x) u=f(x), \quad x \in I, \\
u(0)=u^{\prime}(0)=u^{\prime \prime}(0)=0 .
\end{gathered}
$$

We denote by $x_{N, j}^{(\alpha, \beta)}, 0 \leqslant j \leqslant N$, the nodes of the standard Jacobi-Gauss interpolation on the interval $(-1,1)$. Their corresponding Christoffel numbers are $\varpi_{N, j}^{(\alpha, \beta)}, 0 \leqslant j \leqslant N$. The nodes of the shifted Jacobi-Gauss interpolation on the interval $(0, L)$ are the zeros of $P_{L, N+1}^{(\alpha, \beta)}(x)$, which we denote by $x_{L, N, j^{\prime}}^{(\alpha, \beta)} 0 \leqslant j \leqslant N$. Clearly $x_{L, N, j}^{(\alpha, \beta)}=(L / 2)\left(x_{N, j}^{(\alpha, \beta)}+1\right)$, and their corresponding Christoffel numbers are $\varpi_{L, N, j}^{(\alpha, \beta)}=(L / 2)^{\alpha+\beta+1} \varpi_{N, j}^{(\alpha, \beta)}, 0 \leqslant j \leqslant N$. Let $S_{N}(0, L)$ be 
the set of polynomials of degree at most $N$. Thanks to the property of the standard JacobiGauss quadrature, it follows that for any $\phi \in S_{2 N+1}(0, L)$,

$$
\begin{aligned}
\int_{0}^{L}(L-x)^{\alpha} x^{\beta} \phi(x) d x & =\left(\frac{L}{2}\right)^{\alpha+\beta+1} \int_{-1}^{1}(1-x)^{\alpha}(1+x)^{\beta} \phi\left(\frac{L}{2}(x+1)\right) d x \\
& =\left(\frac{L}{2}\right)^{\alpha+\beta+1} \sum_{j=0}^{N} \varpi_{N, j}^{(\alpha, \beta)} \phi\left(\frac{L}{2}\left(x_{N, j}^{(\alpha, \beta)}+1\right)\right)=\sum_{j=0}^{N} \varpi_{L, N, j}^{(\alpha, \beta)} \phi\left(x_{L, N, j}^{(\alpha, \beta)}\right) .
\end{aligned}
$$

We define the discrete inner product and norm as follows:

$$
(u, v)_{w_{L}^{(\alpha, \beta)}, N}=\sum_{j=0}^{N} u\left(x_{L, N, j}^{(\alpha, \beta)}\right) v\left(x_{L, N, j}^{(\alpha, \beta)}\right) \varpi_{L, N, j^{\prime}}^{(\alpha, \beta)} \quad\|u\|_{w_{L}^{(\alpha, \beta)}, N}=\sqrt{(u, u)_{w_{L}^{(\alpha, \beta)}, N^{\prime}}}
$$

where $x_{L, N, j}^{(\alpha, \beta)}$ and $\varpi_{L, N, j}^{(\alpha, \beta)}$ are the nodes and the corresponding weights of the shifted JacobiGauss-quadrature formula on the interval $(0, L)$, respectively.

Obviously, (see, e.g., formula (2.25) of [12])

$$
(u, v)_{w_{L}^{(\alpha, \beta)}, N}=(u, v)_{w_{L}^{(\alpha, \beta)},} \quad \forall u, v \in S_{2 N-1}
$$

Thus, for any $u \in S_{N}(0, L)$, the norms $\|u\|_{w_{L}^{(\alpha, \beta)}, N}$ and $\|u\|_{w_{L}^{(\alpha, \beta)}}$ coincide.

Associating with this quadrature rule, we denote by $I_{N}^{P_{L}^{(\alpha, \beta)}}$ the shifted Jacobi-Gauss interpolation,

$$
I_{N}^{P_{L}^{(\alpha, \beta)}} u\left(x_{L, N, j}^{(\alpha, \beta)}\right)=u\left(x_{L, N, j}^{(\alpha, \beta)}\right), \quad 0 \leq k \leq N
$$

The pseudospectral Galerkin method for (3.1) is to find $u_{N} \in W_{N}$ such that

$$
\begin{gathered}
\left(u_{N}^{\prime \prime \prime}, v_{N}\right)_{w_{L}^{(\alpha, \beta)}}+\left(\gamma_{1}(x) u_{N^{\prime}}^{\prime \prime}, v_{N}\right)_{w_{L}^{(\alpha, \beta)}, N}+\left(\gamma_{2}(x) u_{N}^{\prime}, v_{N}\right)_{w_{L}^{(\alpha, \beta)}, N} \\
+\left(\gamma_{3}(x) u_{N}, v_{N}\right)_{w_{L}^{(\alpha, \beta)}, N}=\left(f, v_{N}\right)_{w_{L}^{(\alpha, \beta)}, N} \quad \forall v_{N} \in W_{N},
\end{gathered}
$$

where $(u, v)_{w_{L}^{(\alpha, \beta)}, N}$ is the discrete inner product of $u$ and $v$ associated with the shifted JacobiGauss quadrature. 
Hence, by setting

$$
\begin{gathered}
u_{N}=\sum_{k=0}^{N-3} \tilde{a}_{k} \phi_{k}, \quad \overline{\mathbf{a}}=\left(\tilde{a}_{0}, \tilde{a}_{1}, \ldots, \tilde{a}_{N-3}\right)^{T}, \\
\tilde{f}_{k}=\left(f, \phi_{k}\right)_{w_{L}^{(\alpha, \beta)}, N^{\prime}} \quad \overline{\mathbf{f}}=\left(\tilde{f}_{0}, \tilde{f}_{1}, \ldots, \tilde{f}_{N-3}\right)^{T}, \\
\tilde{b}_{i j}=\left(\gamma_{1}(x) \phi_{j}^{\prime \prime}, \phi_{i}\right)_{w_{L}^{(\alpha, \beta)}, N^{\prime}} \quad \tilde{c}_{i j}=\left(\gamma_{2}(x) \phi_{j}^{\prime}, \phi_{i}\right)_{w_{L}^{(\alpha, \beta)}, N^{\prime}} \\
\tilde{d}_{i j}=\left(\gamma_{3}(x) \phi_{j}, \phi_{i}\right)_{w_{L}^{(\alpha, \beta)}, N^{\prime}} \\
\tilde{B}=\left(\tilde{b}_{k j}\right), \quad \tilde{C}=\left(\widetilde{c}_{k j}\right), \quad \tilde{D}=\left(\tilde{d}_{k j}\right), \quad 0 \leq k, j \leq N-3 .
\end{gathered}
$$

Then, the linear system (3.6) becomes

$$
(A+\widetilde{B}+\widetilde{C}+\widetilde{D}) \overline{\mathbf{a}}=\overline{\mathbf{f}}
$$

where $A$ is given in Theorem 2.1.

\section{SJC Method for Nonlinear Third-Order Differential Equations}

In this section, we are interested in solving numerically the nonlinear third-order differential equation:

$$
u^{\prime \prime \prime}(x)=F\left(x, u(x), u^{\prime}(x), u^{\prime \prime}(x)\right),
$$

with initial conditions

$$
u(0)=u^{\prime}(0)=u^{\prime \prime}(0)=0 .
$$

It is well known that one can convert (4.1) into third-order system of first-order initialvalue problems. Methods to solve systems of first-order differential equations are simply generalizations of the methods for a single first-order equation, for example, the classical Runge-Kutta of order four. Another alternative spectral method is to use the shifted Jacobi collocation method to solve (4.1)

$$
u_{N}(x)=\sum_{j=0}^{N} b_{j} \phi_{k}(x)
$$


then, making use of formula (2.7) enables one to express explicitly the derivatives $u^{(i)}(x)$, $(i=0,1,2)$ in terms of the expansion coefficients $b_{j}$. The criterion of spectral shifted Jacobi collocation method for solving approximately (4.1) is to find $u_{N}(x) \in S_{N}(0, L)$ such that

$$
u_{N}^{\prime \prime \prime}\left(x_{L, N, k}^{(\alpha, \beta)}\right)=F\left(x_{L, N, k}^{(\alpha, \beta)}, u_{N}\left(x_{L, N, k}^{(\alpha, \beta)}\right), u_{N}^{\prime}\left(x_{L, N, k}^{(\alpha, \beta)}\right), u_{N}^{\prime \prime}\left(x_{L, N, k}^{(\alpha, \beta)}\right)\right), \quad k=0,1, \ldots, N .
$$

is satisfied exactly at the collocation points $x_{L, N, k}^{(\alpha, \beta)}, k=0,1, \ldots, N$. In other words, we have to collocate (4.4) at the $(N+1)$ shifted Jacobi roots $x_{L, N, k}^{(\alpha, \beta)}$, which immediately yields

$$
\sum_{j=0}^{N} b_{j} \phi_{k}^{\prime \prime \prime}(x)=F\left(x, \sum_{j=0}^{N} b_{j} \phi_{k}(x), \sum_{j=0}^{N} b_{j} \phi_{k}^{\prime}(x), \sum_{j=0}^{N} b_{j} \phi_{k}^{\prime \prime}(x)\right)
$$

This constitutes a system of $(N+1)$ nonlinear algebraic equations in the unknown expansion coefficients $b_{j}(j=0,1, \ldots, N)$, which can be solved by using any standard iteration technique, like Newton's iteration method.

\section{Fifth-Order Differential Equations}

In this section, we consider the fifth-order differential equation of the form:

$$
\begin{aligned}
& u^{(v)}+\gamma_{1} u^{(i v)}+\gamma_{2} u^{\prime \prime \prime}+\gamma_{3} u^{\prime \prime}+\gamma_{4} u^{\prime}+\gamma_{5} u=f(x), \quad x \in I, \\
& u(0)=u^{\prime}(0)=u^{\prime \prime}(0)=u^{\prime \prime \prime}(0)=u^{(i v)}(0)=0 .
\end{aligned}
$$

We define

$$
V_{N}=\left\{v_{N} \in S_{N}(0, L): u(0)=u^{\prime}(0)=u^{\prime \prime}(0)=u^{\prime \prime \prime}(0)=u^{(i v)}(0)=0\right\}
$$

The results for fifth-order differential equations will be given without proofs.

\subsection{SJG Method for Constant Coefficients}

For $\gamma_{1}, \gamma_{2}, \gamma_{3}, \gamma_{4}$, and $\gamma_{5}$ are constants, we consider the following shifted Jacobi-Galerkin procedure for (5.1): Find $u_{N} \in V_{N}$ such that

$$
\begin{gathered}
\left(u_{N}^{(v)}, v_{N}\right)_{w_{L}^{(\alpha, \beta)}}+\gamma_{1}\left(u_{N}^{(i v)}, v_{N}\right)_{w_{L}^{(\alpha, \beta)}}+\gamma_{2}\left(u_{N}^{\prime \prime \prime}, v_{N}\right)_{w_{L}^{(\alpha, \beta)}}+\gamma_{3}\left(u_{N}^{\prime \prime}, v_{N}\right)_{w_{L}^{(\alpha, \beta)}} \\
+\gamma_{4}\left(u_{N}^{\prime}, v_{N}\right)_{w_{L}^{(\alpha, \beta)}}+\gamma_{5}\left(u_{N}, v_{N}\right)_{w_{L}^{(\alpha, \beta)}}=\left(f, v_{N}\right)_{w_{L}^{(\alpha, \beta)}, N^{\prime}} \quad \forall v_{N} \in V_{N} .
\end{gathered}
$$


Now, we choose the basis functions $\Phi_{k}(x)$ to be of the form:

$$
\begin{aligned}
\Phi_{k}(x)=\xi_{k}[ & P_{L, k}^{(\alpha, \beta)}(x)+\widehat{\epsilon}_{k} P_{L, k+1}^{(\alpha, \beta)}(x)+\widehat{\varepsilon}_{k} P_{L, k+2}^{(\alpha, \beta)}(x)+\widehat{\zeta}_{k} P_{L, k+3}^{(\alpha, \beta)}(x) \\
+ & \left.\widehat{\mu}_{k} P_{L, k+4}^{(\alpha, \beta)}(x)+\widehat{v}_{k} P_{L, k+5}^{(\alpha, \beta)}(x)\right], \quad k=0,1, \ldots, N-5,
\end{aligned}
$$

It is not difficult to show that the basis functions $\Phi_{k}(x) \in V_{k+5}$ are given by

$$
\begin{aligned}
\Phi_{k}(x)=\xi_{k}[ & P_{L, k}^{(\alpha, \beta)}(x)+\frac{5(k+1)(2 k+\lambda+2)}{(k+\beta+1)(2 k+\lambda+6)} P_{L, k+1}^{(\alpha, \beta)}(x) \\
& +\frac{10(k+1)_{2}(2 k+\lambda+1)(2 k+\lambda+4)}{(k+\beta+1)_{2}(2 k+\lambda+6)_{2}} P_{L, k+2}^{(\alpha, \beta)}(x) \\
& +\frac{10(k+1)_{3}(2 k+\lambda+1)_{2}}{(k+\beta+1)_{3}(2 k+\lambda+7)_{2}} P_{L, k+3}^{(\alpha, \beta)}(x) \\
& +\frac{5(k+1)_{4}(2 k+\lambda+1)_{3}}{(k+\beta+1)_{4}(2 k+\lambda+6)_{2}(2 k+\lambda+9)} P_{L, k+4}^{(\alpha, \beta)}(x) \\
& \left.+\frac{(k+1)_{5}(2 k+\lambda+1)_{4}}{(k+\beta+1)_{5}(2 k+\lambda+6)_{5}} P_{L, k+5}^{(\alpha, \beta)}(x)\right] .
\end{aligned}
$$

Therefore, for $N \geq 5$, we have

$$
V_{N}=\operatorname{span}\left\{\Phi_{0}, \Phi_{1}, \ldots, \Phi_{N-5}\right\}
$$

It is clear that (5.3) is equivalent to

$$
\begin{aligned}
\left(u_{N}^{(v)},\right. & \left.\Phi_{k}(x)\right)_{w_{L}^{(\alpha, \beta)}}+\gamma_{1}\left(u_{N}^{(i v)}, \Phi_{k}(x)\right)_{w_{L}^{(\alpha, \beta)}}+\gamma_{2}\left(u_{N}^{\prime \prime \prime}, \Phi_{k}(x)\right)_{w_{L}^{(\alpha, \beta)}}+\gamma_{3}\left(u_{N^{\prime}}^{\prime \prime}, \Phi_{k}(x)\right)_{w_{L}^{(\alpha, \beta)}} \\
& +\gamma_{4}\left(u_{N}^{\prime}, \Phi_{k}(x)\right)_{w_{L}^{(\alpha, \beta)}}+\gamma_{5}\left(u_{N}, \Phi_{k}(x)\right)_{w_{L}^{(\alpha, \beta)}} \\
= & \left(f, \Phi_{k}(x)\right)_{w_{L}^{(\alpha, \beta)}, N^{\prime}} \quad k=0,1, \ldots, N-5 .
\end{aligned}
$$


Let us denote

$$
\begin{array}{cc}
f_{k}=\left(f, \Phi_{k}(x)\right)_{w_{L}^{(\alpha, \beta)}, N^{\prime}} & \mathbf{f}=\left(f_{0}, f_{1}, \ldots, f_{N-5}\right)^{T}, \\
u_{N}(x)=\sum_{n=0}^{N-5} v_{n} \Phi_{n}(x), & \mathbf{v}=\left(v_{0}, v_{1}, \ldots, v_{N-5}\right)^{T} . \\
r_{i j}=\left(\Phi_{j}^{(v)}, \Phi_{i}\right)_{w_{L}^{(\alpha, \beta)},} & q_{i j}=\left(\Phi_{j}^{(i v)}, \Phi_{i}\right)_{w_{L}^{(\alpha, \beta)},} \\
y_{i j}=\left(\Phi_{j}^{\prime \prime \prime}, \Phi_{i}\right)_{w_{L}^{(\alpha, \beta)},} & s_{i j}=\left(\Phi_{j}^{\prime \prime}, \Phi_{i}\right)_{w_{L}^{(\alpha, \beta)},}, \\
t_{i j}=\left(\Phi_{j}^{\prime}, \Phi_{i}\right)_{w_{L}^{(\alpha, \beta)},} & u_{i j}=\left(\Phi_{j}, \Phi_{i}\right)_{w_{L}^{(\alpha, \beta)},}
\end{array}
$$

then equation (5.7) is equivalent to the following matrix equation:

$$
\left(R+\gamma_{1} Q+\gamma_{2} Y+\gamma_{3} S+\gamma_{4} T+\gamma_{5} U\right) \mathbf{v}=\mathbf{f}
$$

where the nonzero elements of the matrices $R, Q, Y, S, T$, and $U$ are given explicitly in the following theorem.

Theorem 5.1. If one takes $\Phi_{k}(x)$ as defined in (5.4), and if one denotes $p_{k j}=\left(\Phi_{j}^{(v)}(x), \Phi_{k}(x)\right)_{w_{L}^{(\alpha, \beta)}}$, $q_{k j}=\left(\Phi_{j}^{(i v)}(x), \Phi_{k}(x)\right)_{w_{L}^{(\alpha, \beta)},} y_{k j}=\left(\Phi_{j}^{\prime \prime \prime}(x), \Phi_{k}(x)\right)_{w_{L}^{(\alpha, \beta)}}, s_{k j}=\left(\Phi_{j}^{\prime \prime}(x), \Phi_{k}(x)\right)_{w_{L}^{(\alpha, \beta)},} t_{k j}=\left(\Phi_{j}^{\prime}(x)\right.$,

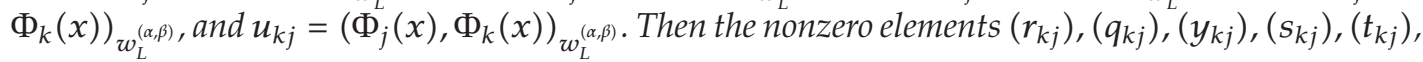
and $\left(u_{k j}\right)$ for $0 \leq k, j \leq N-5$ are given as follows:

$$
\begin{aligned}
r_{k k}= & \frac{L^{\alpha+\beta-4}(2 k+\lambda+1)_{5}(2 k+\lambda+1)_{4} \Gamma(k+6)(\Gamma(\alpha+1))^{2} \Gamma(k+\beta+1)}{(k+\beta+1)_{5} \Gamma(k+\alpha+1) \Gamma(k+\lambda+5)}, \\
r_{k j}=\xi_{k} \xi_{j}[ & O_{5}(j, k, \alpha, \beta) h_{L, k}^{(\alpha, \beta)}+O_{5}(j, k+1, \alpha, \beta) \widehat{\epsilon}_{k} h_{L, k+1}^{(\alpha, \beta)}+O_{5}(j, k+2, \alpha, \beta) \widehat{\varepsilon}_{k} h_{L, k+2}^{(\alpha, \beta)} \\
& +O_{5}(j, k+3, \alpha, \beta) \widehat{\zeta}_{k} h_{L, k+3}^{(\alpha, \beta)}+O_{5}(j, k+4, \alpha, \beta) \widehat{\mu}_{k} h_{L, k+4}^{(\alpha, \beta)} \\
& \left.+O_{5}(j, k+5, \alpha, \beta) \widehat{v}_{k} h_{L, k+5}^{(\alpha, \beta)}\right], \quad j=k+n, n \geq 1, \\
q_{k j}=\xi_{k} \xi_{j}[ & O_{4}(j, k, \alpha, \beta) h_{L, k}^{(\alpha, \beta)}+O_{4}(j, k+1, \alpha, \beta) \widehat{\epsilon}_{k} h_{L, k+1}^{(\alpha, \beta)}+O_{4}(j, k+2, \alpha, \beta) \widehat{\varepsilon}_{k} h_{L, k+2}^{(\alpha, \beta)} \\
& +O_{4}(j, k+3, \alpha, \beta) \widehat{\zeta}_{k} h_{L, k+3}^{(\alpha, \beta)}+O_{4}(j, k+4, \alpha, \beta) \widehat{\mu}_{k} h_{L, k+4}^{(\alpha, \beta)} \\
& \left.+O_{4}(j, k+5, \alpha, \beta) \widehat{v}_{k} h_{L, k+5}^{(\alpha, \beta)}\right], \quad j=k+n-1, n \geq 0,
\end{aligned}
$$




$$
\begin{aligned}
& y_{k j}=\xi_{k} \xi_{j}\left[O_{3}(j, k, \alpha, \beta) h_{L, k}^{(\alpha, \beta)}+O_{3}(j, k+1, \alpha, \beta) \widehat{\epsilon}_{k} h_{L, k+1}^{(\alpha, \beta)}+O_{3}(j, k+2, \alpha, \beta) \widehat{\varepsilon}_{k} h_{L, k+2}^{(\alpha, \beta)}\right. \\
& +O_{3}(j, k+3, \alpha, \beta) \widehat{\zeta}_{k} h_{L, k+3}^{(\alpha, \beta)}+O_{3}(j, k+4, \alpha, \beta) \widehat{\mu}_{k} h_{L, k+4}^{(\alpha, \beta)} \\
& \left.+O_{3}(j, k+5, \alpha, \beta) \widehat{v}_{k} h_{L, k+5}^{(\alpha, \beta)}\right], \quad j=k+n-2, n \geq 0, \\
& s_{k j}=\xi_{k} \xi_{j}\left[O_{2}(j, k, \alpha, \beta) h_{L, k}^{(\alpha, \beta)}+O_{2}(j, k+1, \alpha, \beta) \widehat{\epsilon}_{k} h_{L, k+1}^{(\alpha, \beta)}+O_{2}(j, k+2, \alpha, \beta) \widehat{\varepsilon}_{k} h_{L, k+2}^{(\alpha, \beta)}\right. \\
& +O_{2}(j, k+3, \alpha, \beta) \widehat{\zeta}_{k} h_{L, k+3}^{(\alpha, \beta)}+O_{2}(j, k+4, \alpha, \beta) \widehat{\mu}_{k} h_{L, k+4}^{(\alpha, \beta)} \\
& \left.+O_{2}(j, k+5, \alpha, \beta) \widehat{v}_{k} h_{L, k+5}^{(\alpha, \beta)}\right], \quad j=k+n-3, n \geq 0, \\
& t_{k j}=\xi_{k} \xi_{j}\left[O_{1}(j, k, \alpha, \beta) h_{L, k}^{(\alpha, \beta)}+O_{1}(j, k+1, \alpha, \beta) \widehat{\epsilon}_{k} h_{L, k+1}^{(\alpha, \beta)}+O_{1}(j, k+2, \alpha, \beta) \widehat{\varepsilon}_{k} h_{L, k+2}^{(\alpha, \beta)}\right. \\
& +O_{1}(j, k+3, \alpha, \beta) \widehat{\zeta}_{k} h_{L, k+3}^{(\alpha, \beta)}+O_{1}(j, k+4, \alpha, \beta) \widehat{\mu}_{k} h_{L, k+4}^{(\alpha, \beta)} \\
& \left.+O_{1}(j, k+5, \alpha, \beta) \widehat{v}_{k} h_{L, k+5}^{(\alpha, \beta)}\right], \quad j=k+n-4, n \geq 0, \\
& u_{k k}=\xi_{k}^{2}\left[h_{L, k}^{(\alpha, \beta)}+\widehat{\epsilon}_{k} \widehat{\epsilon}_{k} h_{L, k+1}^{(\alpha, \beta)}+\widehat{\varepsilon}_{k} \widehat{\varepsilon}_{k} h_{L, k+2}^{(\alpha, \beta)}+\widehat{\zeta}_{k} \widehat{\zeta}_{k} h_{L, k+3}^{(\alpha, \beta)}+\widehat{\mu}_{k} \widehat{\mu}_{k} h_{L, k+4}^{(\alpha, \beta)}+\widehat{v}_{k} \widehat{v}_{k} h_{L, k+5}^{(\alpha, \beta)}\right], \\
& u_{k+1, k}=u_{k, k+1}=\xi_{k} \xi_{k+1}\left[\widehat{\epsilon}_{k} h_{L, k+1}^{(\alpha, \beta)}+\widehat{\epsilon}_{k+1} \widehat{\varepsilon}_{k} h_{L, k+2}^{(\alpha, \beta)}+\widehat{\varepsilon}_{k+1} \widehat{\zeta}_{k} h_{L, k+3}^{(\alpha, \beta)}+\widehat{\zeta}_{k+1} \widehat{\mu}_{k} h_{L, k+4}^{(\alpha, \beta)}+\widehat{\mu}_{k+1} \widehat{v}_{k} h_{L, k+5}^{(\alpha, \beta)}\right] \text {, } \\
& u_{k+2, k}=u_{k, k+2}=\xi_{k} \xi_{k+2}\left[\widehat{\varepsilon}_{k} h_{L, k+2}^{(\alpha, \beta)}+\widehat{\epsilon}_{k+2} \widehat{\zeta}_{k} h_{L, k+3}^{(\alpha, \beta)}+\widehat{\varepsilon}_{k+2} \widehat{\mu}_{k} h_{L, k+4}^{(\alpha, \beta)}+\widehat{\zeta}_{k+2} \widehat{v}_{k} h_{L, k+5}^{(\alpha, \beta)}\right] \\
& u_{k+3, k}=u_{k, k+3}=\xi_{k} \xi_{k+3}\left[\widehat{\zeta}_{k} h_{L, k+3}^{(\alpha, \beta)}+\widehat{\epsilon}_{k+3} \widehat{\mu}_{k} h_{L, k+4}^{(\alpha, \beta)}+\widehat{\varepsilon}_{k+3} \widehat{v}_{k} h_{L, k+5}^{(\alpha, \beta)}\right] \text {, } \\
& u_{k+4, k}=u_{k, k+4}=\xi_{k} \xi_{k+4}\left[\widehat{\mu}_{k} h_{L, k+4}^{(\alpha, \beta)}+\widehat{\epsilon}_{k+4} \widehat{v}_{k} h_{L, k+5}^{(\alpha, \beta)}\right], \quad u_{k+5, k}=u_{k, k+5}=\xi_{k} \xi_{k+5} \widehat{v}_{k} h_{L, k+5}^{(\alpha, \beta)}
\end{aligned}
$$

where

$$
\begin{aligned}
O_{i}(j, k, \alpha, \beta)= & C_{i}(j, k, \alpha, \beta)+\widehat{\epsilon}_{j} C_{i}(j+1, k, \alpha, \beta)+\widehat{\varepsilon}_{j} C_{i}(j+2, k, \alpha, \beta) \\
& +\widehat{\zeta}_{j} C_{i}(j+3, k, \alpha, \beta)+\widehat{\mu}_{j} C_{i}(j+4, k, \alpha, \beta)+\widehat{v}_{j} C_{i}(j+5, k, \alpha, \beta) .
\end{aligned}
$$

Proof. The proof of this theorem is not difficult, and it can be accomplished by following the same procedure used in proving Theorem 2.1. 
In the following, we can always modify the right-hand side to take care of the nonhomogeneous initial conditions. Let us consider for instance the one-dimensional fifth-order differential equation (5.1) subject to the nonhomogeneous initial conditions:

$$
\begin{gathered}
u(0)=a_{+}, \quad u^{\prime}(0)=a_{-}, \quad u^{\prime \prime}(0)=\tilde{a}_{+}, \\
u^{\prime \prime \prime}(0)=\tilde{a}_{-}, \quad u^{(i v)}(0)=b_{+} .
\end{gathered}
$$

We proceed as follows.

Set

$$
V(x)=u(x)+b_{0}+b_{1} x+b_{2} x^{2}+b_{3} x^{3}+b_{4} x^{4},
$$

where

$$
b_{0}=-a_{+}, \quad b_{1}=-a_{-}, \quad b_{2}=\frac{-\tilde{a}_{+}}{2}, \quad b_{3}=\frac{-\tilde{a}_{-}}{6}, \quad b_{4}=\frac{-b_{+}}{24} .
$$

The transformation (5.13) turns the nonhomogeneous initial conditions (5.12) into the homogeneous initial conditions:

$$
V(0)=V^{\prime}(0)=V^{\prime \prime}(0)=V^{\prime \prime \prime}(0)=V^{(i v)}(0)=0 .
$$

Hence, it suffices to solve the following modified one-dimensional fifth-order equation:

$$
V^{(v)}+\gamma_{1} V^{(i v)}+\gamma_{2} V^{\prime \prime \prime}+\gamma_{3} V^{\prime \prime}+\gamma_{4} V^{\prime}+\gamma_{5} V=f^{*}(x) \text { in } I=(0, L),
$$

subject to the homogeneous initial conditions (5.15), where $V(x)$ is given by (5.13), and

$$
\begin{aligned}
f^{*}(x)= & f(x)-\left(24 \gamma_{1} b_{4}+6 \gamma_{2} b_{3}+2 \gamma_{3} b_{2}+\gamma_{4} b_{1}+\gamma_{5} b_{0}\right)-\left(24 \gamma_{2} b_{4}+6 \gamma_{3} b_{3}+2 \gamma_{4} b_{2}+\gamma_{5} b_{1}\right) x \\
& -\left(12 \gamma_{3} b_{4}+3 \gamma_{4} b_{3}+\gamma_{5} b_{2}\right) x^{2}-\left(4 \gamma_{4} b_{4}+\gamma_{5} b_{3}\right) x^{3}-\gamma_{5} b_{4} x^{4} .
\end{aligned}
$$

\subsection{Fifth-Order Equations with Variable Coefficients}

Let us consider the fifth-order differential equation (5.1) with $\gamma_{1}, \gamma_{2}, \gamma_{3}, \gamma_{4}$, and $\gamma_{5}$ are variables. The pseudospectral Galerkin method for (5.1) is to find $u_{N} \in V_{N}$ such that

$$
\begin{aligned}
\left(u_{N}^{(v)},\right. & \left.v_{N}\right)_{w_{L}^{(\alpha, \beta)}}+\left(\gamma_{1}(x) u_{N}^{(i v)}, v_{N}\right)_{w_{L}^{(\alpha, \beta)}, N}+\left(\gamma_{2}(x) u_{N}^{\prime \prime \prime}, v_{N}\right)_{w_{L}^{(\alpha, \beta)}, N} \\
& +\left(\gamma_{3}(x) u_{N}^{\prime \prime}, v_{N}\right)_{w_{L}^{(\alpha, \beta)}, N}+\left(\gamma_{4}(x) u_{N}^{\prime}, v_{N}\right)_{w_{L}^{(\alpha, \beta)}, N}+\left(\gamma_{5}(x) u_{N}, v_{N}\right)_{w_{L}^{(\alpha, \beta)}, N} \\
& =\left(f, v_{N}\right)_{w_{L}^{(\alpha, \beta)}, N} \quad \forall v_{N} \in V_{N},
\end{aligned}
$$


where $(u, v)_{w_{L}^{(\alpha, \beta)}, N}$ is the discrete inner product of $u$ and $v$ associated with the shifted JacobiGauss quadrature (for details, see Section 3).

\subsection{Nonlinear Fifth-Order Differential Equations}

In this section, we are interested in solving numerically the nonlinear fifth-order differential equation:

$$
u^{(v)}(x)=F\left(x, u(x), u^{\prime}(x), u^{\prime \prime}(x), u^{\prime \prime \prime}(x), u^{(i v)}(x)\right)
$$

with initial conditions:

$$
u(0)=u^{\prime}(0)=u^{\prime \prime}(0)=u^{\prime \prime \prime}(0)=u^{(i v)}(0)=0
$$

It is well known that one can convert (5.19) into fifth-order system of first-order initialvalue problems. Methods to solve systems of first-order differential equations are simply generalizations of the methods for a single first-order equation, for example, the classical Runge-Kutta of order four. Another alternative spectral method is to use the shifted Jacobi collocation method to solve (5.19):

$$
u_{N}(x)=\sum_{j=0}^{N} b_{j} \Phi_{k}(x)
$$

Then, making use of formula (2.7) enables one to express explicitly the derivatives $u^{(i)}(x)$, $(i=0,1,2,3,4)$ in terms of the expansion coefficients $b_{j}$. The criterion of spectral shifted Jacobi collocation method for solving approximately (5.19) is to find $u_{N}(x) \in S_{N}(0, L)$ such that

$$
\begin{array}{r}
u_{N}^{(v)}\left(x_{L, N, k}^{(\alpha, \beta)}\right)=F\left(x_{L, N, k}^{(\alpha, \beta)}, u_{N}\left(x_{L, N, k}^{(\alpha, \beta)}\right), u_{N}^{\prime}\left(x_{L, N, k}^{(\alpha, \beta)}\right), u_{N}^{\prime \prime}\left(x_{L, N, k}^{(\alpha, \beta)}\right), u_{N}^{\prime \prime \prime}\left(x_{L, N, k}^{(\alpha, \beta)}\right), u_{N}^{(i v)}\left(x_{L, N, k}^{(\alpha, \beta)}\right)\right), \\
k=0,1, \ldots, N,
\end{array}
$$

is satisfied exactly at the collocation points $x_{L, N, k}^{(\alpha, \beta)}, k=0,1, \ldots, N$. In other words, we have to collocate (5.22) at the $(N+1)$ shifted Jacobi roots $x_{L, N, k}^{(\alpha, \beta)}$, which immediately yields

$$
\sum_{j=0}^{N} b_{j} \Phi_{k}^{(v)}(x)=F\left(x, \sum_{j=0}^{N} b_{j} \Phi_{k}(x), \sum_{j=0}^{N} b_{j} \Phi_{k}^{\prime}(x), \sum_{j=0}^{N} b_{j} \Phi_{k}^{\prime \prime}(x), \sum_{j=0}^{N} b_{j} \Phi_{k}^{\prime \prime \prime}(x), \sum_{j=0}^{N} b_{j} \Phi_{k}^{(i v)}(x)\right)
$$

This constitute a system of $(N+1)$ nonlinear algebraic equations in the unknown expansion coefficients $b_{j}(j=0,1, \ldots, N)$, which can be solved by using any standard iteration technique, like Newton's iteration method. 
Table 1: Maximum pointwise error using SJG method for $N=8,16,24$ for Example 6.1.

\begin{tabular}{|c|c|c|c|c|c|c|}
\hline$N$ & $\alpha$ & $\beta$ & SJG & $\alpha$ & $\beta$ & SJG \\
\hline 8 & \multirow{3}{*}{$\frac{1}{2}$} & \multirow{3}{*}{$\frac{1}{2}$} & $1.542 \cdot 10^{-1}$ & \multirow{3}{*}{$\frac{1}{2}$} & \multirow{3}{*}{$-\frac{1}{2}$} & $8.093 \cdot 10^{-2}$ \\
\hline 16 & & & $5.513 \cdot 10^{-8}$ & & & $1.642 \cdot 10^{-8}$ \\
\hline 24 & & & $1.598 \cdot 10^{-14}$ & & & $4.707 \cdot 10^{-14}$ \\
\hline 8 & \multirow{3}{*}{$-\frac{1}{2}$} & \multirow{3}{*}{$\frac{1}{2}$} & $2.581 \cdot 10^{-1}$ & \multirow{3}{*}{$-\frac{1}{2}$} & \multirow{3}{*}{$-\frac{1}{2}$} & $1.417 \cdot 10^{-1}$ \\
\hline 16 & & & $9.369 \cdot 10^{-8}$ & & & $2.875 \cdot 10^{-8}$ \\
\hline 24 & & & $1.509 \cdot 10^{-14}$ & & & $2.575 \cdot 10^{-14}$ \\
\hline
\end{tabular}

\section{Numerical Results}

To illustrate the effectiveness of the proposed methods in the present paper, several test examples are carried out in this section. Comparisons of the results obtained by the present methods with those obtained by other methods reveal that the present methods are very efficient and more robust.

Example 6.1. Consider the linear third-order problem (see [23]):

$$
u^{\prime \prime \prime}(x)+2 u^{\prime \prime}(x)-u^{\prime}(x)-2 u(x)=f(x), \quad x \in[0,3],
$$

subject to the initial condition:

$$
u(0)=1, \quad u^{\prime}(0)=2, \quad u^{\prime \prime}(0)=0,
$$

where $f$ is selected such that exact solution is

$$
u(x)=\frac{1}{36}(6 x-5) e^{x}-\frac{4}{9} e^{-2 x}+\frac{1}{4} e^{-x}+\frac{4}{3} e^{x} .
$$

Table 1 list the maximum pointwise error of $u-u_{N}$ using the SJG method with various choices of $N$. Numerical results of this problem show that the SJG method converges exponentially.

Example 6.2. Consider the linear third-order problem with variable coefficients:

$$
u^{\prime \prime \prime}-\cos (4 x) u^{\prime \prime}-e^{3 x} u^{\prime}+\left(\sin (x)+x^{3}\right) u=f(x), \quad x \in[0,3]
$$

subject to the initial condition:

$$
u(0)=1, \quad u^{\prime}(0)=2, \quad u^{\prime \prime}(0)=0,
$$

where $f$ is selected such that exact solution is

$$
u(x)=\frac{1}{36}(6 x-5) e^{x}-\frac{4}{9} e^{-2 x}+\frac{1}{4} e^{-x}+\frac{4}{3} e^{x} .
$$


Table 2: Maximum pointwise error using P-SJG method for $N=8,16,24$ for Example 6.2.

\begin{tabular}{lcccccc}
\hline$N$ & $\alpha$ & $\beta$ & P-SJG & $\alpha$ & $\beta$ & P-SJG \\
\hline 8 & & $7.346 \cdot 10^{-4}$ & & & $6.848 \cdot 10^{-4}$ \\
16 & 1 & 1 & $3.173 \cdot 10^{-11}$ & $\frac{1}{2}$ & $\frac{1}{2}$ & $2.659 \cdot 10^{-11}$ \\
24 & & $1.533 \cdot 10^{-12}$ & $6.366 \cdot 10^{-4}$ & & $1.056 \cdot 10^{-12}$ \\
\hline 8 & & $1.363 \cdot 10^{-11}$ & $-\frac{3}{4}$ & $-\frac{3}{4}$ & $4.459 \cdot 10^{-4}$ \\
16 & 0 & 0 & $2.318 \cdot 10^{-12}$ & & & $5.574 \cdot 10^{-12}$ \\
24 & & & & & & $2.626 \cdot 10^{-12}$ \\
\hline
\end{tabular}

Table 3: Maximum pointwise error using SJG method for $N=8,16,24$ for Example 6.3.

\begin{tabular}{lcccccc}
\hline$N$ & $\alpha$ & $\beta$ & SJG & $\alpha$ & $\beta$ & SJG \\
\hline 8 & 1 & $\frac{1}{2}$ & $9.742 \cdot 10^{-2}$ & & $1.782 \cdot 10^{-2}$ \\
16 & $\frac{1}{2}$ & $2.220 \cdot 10^{-15}$ & $\frac{1}{2}$ & $-\frac{1}{2}$ & $4.319 \cdot 10^{-11}$ \\
24 & & $6.011 \cdot 10^{-2}$ & & $1.998 \cdot 10^{-15}$ \\
\hline 8 & $-\frac{1}{2}$ & $\frac{1}{2}$ & $1.755 \cdot 10^{-10}$ & $-\frac{1}{2}$ & $-\frac{1}{2}$ & $8.691 \cdot 10^{-2}$ \\
16 & & $1.443 \cdot 10^{-15}$ & & & $1.095 \cdot 10^{-11}$ \\
24 & & & & & \\
\hline
\end{tabular}

Table 2 list the Maximum pointwise error, using the P-SJG method with various choices of $\alpha, \beta$, and $N$. Numerical results of third-order differential equation with variable coefficients show that the P-SJG method converges exponentially.

Example 6.3. Consider the linear fifth-order problem (see [24]):

$$
u^{(v)}(x)-32 u(x)=f(x), \quad x \in[0,1]
$$

subject to the initial condition:

$$
u(0)=1, \quad u^{\prime}(0)=3, \quad u^{\prime \prime}(0)=4, \quad u^{\prime \prime \prime}(0)=7, \quad u^{(i v)}(0)=16,
$$

where $f$ is selected such that exact solution is

$$
u(x)=e^{2 x}+\sin x .
$$

Table 3 list the maximum absolute error of $u-u_{N}$ using the SJG method with various choices of $\alpha, \beta$, and $N$. It is seen that for the given initial conditions, the maximum absolute error in the He's variational iteration method [24] with eight iterations is $5.579 \cdot 10^{-4}$ (see, Table 2 in [24]).

Example 6.4. Consider the linear fifth-order problem with variable coefficients:

$$
u^{(v)}-\sin (3 x) u^{(i v)}-e^{2 x} u^{\prime \prime \prime}-\left(\cos (x)+x^{2}\right) u^{\prime \prime}-x \sin x u^{\prime}+x^{2} u=f(x), \quad x \in[0,2],
$$


Table 4: Maximum pointwise error using P-SJG method for $N=8,16,24$ for Example 6.4.

\begin{tabular}{|c|c|c|c|c|c|c|}
\hline$N$ & $\alpha$ & $\beta$ & P-SJG & $\alpha$ & $\beta$ & P-SJG \\
\hline 8 & \multirow{3}{*}{$\frac{1}{2}$} & \multirow{3}{*}{$\frac{1}{2}$} & $9.036 \cdot 10^{-3}$ & \multirow{3}{*}{$\frac{1}{2}$} & \multirow{3}{*}{$-\frac{1}{2}$} & $7.005 \cdot 10^{-3}$ \\
\hline 16 & & & $1.546 \cdot 10^{-10}$ & & & $1.694 \cdot 10^{-10}$ \\
\hline 24 & & & $2.673 \cdot 10^{-13}$ & & & $1.687 \cdot 10^{-13}$ \\
\hline 8 & \multirow{3}{*}{$-\frac{1}{2}$} & \multirow{3}{*}{$\frac{1}{2}$} & $1.350 \cdot 10^{-2}$ & \multirow{3}{*}{$-\frac{1}{2}$} & \multirow{3}{*}{$-\frac{1}{2}$} & $1.100 \cdot 10^{-2}$ \\
\hline 16 & & & $1.280 \cdot 10^{-10}$ & & & $1.234 \cdot 10^{-10}$ \\
\hline 24 & & & $1.243 \cdot 10^{-14}$ & & & $9.858 \cdot 10^{-14}$ \\
\hline
\end{tabular}

subject to the initial condition:

$$
u(0)=1, \quad u^{\prime}(0)=1, \quad u^{\prime \prime}(0)=0, \quad u^{\prime \prime \prime}(0)=4, \quad u^{(i v)}(0)=-4,
$$

where $f$ is selected such that exact solution is

$$
u(x)=x^{2} \sin x+e^{x} \cos x
$$

Table 4 list the Maximum pointwise error, using the P-SJG method with various choices of $\alpha, \beta$, and $N$. Numerical results of fifth-order differential equation with variable coefficients show that the P-SJG method converges exponentially.

Example 6.5. We consider in this example the third order nonlinear problem:

$$
u^{(3)}(x)+\frac{2}{x} u^{(2)}(x)+u(x)^{2}=\left(14-x^{2}\right) \cos x+\frac{\sin x\left(4-8 x^{2}+x^{5} \sin x\right)}{x}, \quad x \in[0,1]
$$

with initial conditions given at three different points,

$$
u(0)=u^{(1)}(0)=u^{(2)}(0)=0 .
$$

The exact solution of this problem is

$$
u(x)=x^{2} \sin x
$$

In Table 5, we list the absolute errors obtained by the shifted Jacobi collocation method, with different values of $\alpha, \beta$ and at $N=14$.

Example 6.6. We consider in this example the fifth order nonlinear problem:

$$
u^{(5)}(x)+u(x) u^{(4)}(x)=f(x), \quad x \in[0,1]
$$


Table 5: Absolute error using SJC method for $N=14$ Example 6.5.

\begin{tabular}{lccc}
\hline$x$ & $\alpha=-1 / 2, \beta=1 / 2$ & $\alpha=0, \beta=0$ & $\alpha=1 / 2, \beta=-1 / 2$ \\
\hline 0.0 & $3.613 \cdot 10^{-17}$ & $6.918 \cdot 10^{-18}$ & $8.474 \cdot 10^{-17}$ \\
0.1 & $1.192 \cdot 10^{-17}$ & $4.553 \cdot 10^{-18}$ & $2.936 \cdot 10^{-16}$ \\
0.2 & $2.081 \cdot 10^{-17}$ & $1.387 \cdot 10^{-17}$ & $3.330 \cdot 10^{-16}$ \\
0.3 & $3.469 \cdot 10^{-18}$ & $5.551 \cdot 10^{-17}$ & $3.469 \cdot 10^{-18}$ \\
0.4 & $1.387 \cdot 10^{-17}$ & $6.938 \cdot 10^{-18}$ & $2.081 \cdot 10^{-17}$ \\
0.5 & $4.163 \cdot 10^{-17}$ & $4.163 \cdot 10^{-17}$ & $1.387 \cdot 10^{-17}$ \\
0.6 & $1.110 \cdot 10^{-16}$ & $1.110 \cdot 10^{-16}$ & $5.551 \cdot 10^{-17}$ \\
0.7 & $5.551 \cdot 10^{-17}$ & $1.110 \cdot 10^{-16}$ & $5.551 \cdot 10^{-17}$ \\
0.8 & $5.551 \cdot 10^{-17}$ & $5.551 \cdot 10^{-17}$ & $1.110 \cdot 10^{-16}$ \\
0.9 & 0 & $1.110 \cdot 10^{-16}$ & $1.110 \cdot 10^{-16}$ \\
1.0 & 0 & $1.110 \cdot 10^{-16}$ & $1.110 \cdot 10^{-16}$ \\
\hline
\end{tabular}

Table 6: Absolute error using SJC method for $N=18$ Example 6.6.

\begin{tabular}{lccc}
\hline$x$ & $\alpha=-1 / 2, \beta=1 / 2$ & $\alpha=0, \beta=0$ & $\alpha=1 / 2, \beta=-1 / 2$ \\
\hline 0.0 & 0 & $1.991 \cdot 10^{-18}$ & $6.938 \cdot 10^{-18}$ \\
0.1 & $1.665 \cdot 10^{-16}$ & $3.037 \cdot 10^{-17}$ & $2.638 \cdot 10^{-16}$ \\
0.2 & $4.996 \cdot 10^{-16}$ & $4.353 \cdot 10^{-17}$ & $3.538 \cdot 10^{-16}$ \\
0.3 & $1.665 \cdot 10^{-16}$ & $1.908 \cdot 10^{-17}$ & $3.764 \cdot 10^{-16}$ \\
0.4 & $1.665 \cdot 10^{-16}$ & $6.418 \cdot 10^{-17}$ & $3.122 \cdot 10^{-17}$ \\
0.5 & $2.775 \cdot 10^{-17}$ & $5.204 \cdot 10^{-17}$ & $6.938 \cdot 10^{-18}$ \\
0.6 & 0 & $8.326 \cdot 10^{-17}$ & $5.204 \cdot 10^{-17}$ \\
0.7 & $1.110 \cdot 10^{-16}$ & $1.110 \cdot 10^{-16}$ & $7.632 \cdot 10^{-17}$ \\
0.8 & $1.665 \cdot 10^{-16}$ & $1.110 \cdot 10^{-16}$ & $4.163 \cdot 10^{-17}$ \\
0.9 & $2.775 \cdot 10^{-16}$ & $1.110 \cdot 10^{-16}$ & $1.387 \cdot 10^{-16}$ \\
1.0 & $5.551 \cdot 10^{-16}$ & $1.110 \cdot 10^{-16}$ & $2.220 \cdot 10^{-16}$ \\
\hline
\end{tabular}

with initial conditions given at three different points,

$$
u(0)=u^{(1)}(0)=u^{(2)}(0)=u^{(3)}(0)=u^{(4)}(0)=0 .
$$

The exact solution of this problem is

$$
u(x)=x^{5} \cos x
$$

In Table 6, we list the absolute errors obtained by the shifted Jacobi collocation method, with different values of $\alpha, \beta$ and at $N=18$.

\section{Concluding Remarks}

In this paper, we described a shifted Jacobi Galerkin method for third- and fifth-order ODEs with constant coefficients subject to homogeneous and nonhomogeneous initial conditions. The initial boundary conditions are satisfied exactly by expanding the unknown variable into a polynomial basis of functions which are built upon the shifted Jacobi polynomials. 
Because of the constant coefficients, the matrix elements of the discrete operators are provided explicitly, and this in turn greatly simplifies the steps and the computational effort for obtaining solutions. We have also presented some efficient direct solvers for the same equations with variable coefficients using P-SJG method.

An efficient and accurate numerical scheme based on the SJC spectral method is proposed for solving these equations. The problem is reduced to the solution of nonlinear algebraic equations. Through several numerical examples, we evaluate the accuracy and performance of the proposed algorithms. The algorithms are easy to implement and yield very accurate results.

\section{Acknowledgments}

This paper was funded by the Deanship of Scientific Research (DSR), King Abdulaziz University, Jeddah. The authors, therefore, acknowledge with thanks DSR technical and financial support. Also, they would like to thank the editor and the reviewers for their constructive comments and suggestions to improve the quality of the paper.

\section{References}

[1] J. P. Boyd, Chebyshev and Fourier Spectral Methods, Dover, Mineola, NY, USA, 2nd edition, 2001.

[2] C. Canuto, M. Y. Hussaini, A. Quarteroni, and T. A. Zang, Spectral Methods in Fluid Dynamics, Springer, New York, NY, USA, 1989.

[3] B.-Y. Guo, Spectral Methods and Their Applications, World Scientific, River Edge, NJ, USA, 1998.

[4] A. H. Bhrawy, A. S. Alofi, and S. I. El-Soubhy, "An extension of the legendre-galerkin method for solving sixth-order differential equations with variable polynomial coefficients," Mathematical Problems in Engineering, vol. 2012, Article ID 896575, 13 pages, 2012.

[5] E. H. Doha, W. M. Abd-Elhameed, and A. H. Bhrawy, "Efficient spectral ultraspherical-Galerkin algorithms for the direct solution of $2 n$ th-order linear differential equations," Applied Mathematical Modelling, vol. 33, no. 4, pp. 1982-1996, 2009.

[6] E. H. Doha and A. H. Bhrawy, "Efficient spectral-Galerkin algorithms for direct solution for secondorder differential equations using Jacobi polynomials," Numerical Algorithms, vol. 42, no. 2, pp. 137$164,2006$.

[7] E. H. Doha and A. H. Bhrawy, "Efficient spectral-Galerkin algorithms for direct solution of the integrated forms of second-order equations using ultraspherical polynomials," The ANZIAM Journal, vol. 48, no. 3, pp. 361-386, 2007.

[8] E. H. Doha and A. H. Bhrawy, "A Jacobi spectral Galerkin method for the integrated forms of fourthorder elliptic differential equations," Numerical Methods for Partial Differential Equations, vol. 25, no. 3, pp. 712-739, 2009.

[9] E. H. Doha, A. H. Bhrawy, and W. M. Abd-Elhameed, "Jacobi spectral Galerkin method for elliptic Neumann problems," Numerical Algorithms, vol. 50, no. 1, pp. 67-91, 2009.

[10] E. H. Doha and W. M. Abd-Elhameed, "Efficient solutions of multidimensional sixth-order boundary value problems using symmetric generalized Jacobi-Galerkin method," Abstract and Applied Analysis, Abstract and Applied Analysis, vol. 2012, Article ID 749370, 19 pages, 2012.

[11] E. H. Doha and A. H. Bhrawy, "An efficient direct solver for multidimensional elliptic Robin boundary value problems using a Legendre spectral-Galerkin method," Computers and Mathematics with Applications, vol. 64, no. 4, pp. 558-571, 2012.

[12] C. Lanczos, Applied Analysis, Pitman, London, UK, 1957.

[13] A. H. Bhrawy, A. S. Alofi, and S. I. El-Soubhy, "Spectral shifted Jacobi tau and collocation methods for solving fifth-order boundary value problems," Abstract and Applied Analysis, vol. 2011, Article ID 823273, 14 pages, 2011.

[14] E. H. Doha, A. H. Bhrawy, and R. M. Hafez, “On shifted Jacobi spectral method for high-order multipoint boundary value problems," Communications in Nonlinear Science and Numerical Simulation, vol. 17, pp. 3802-3810, 2012. 
[15] A. H. Bhrawy and W. M. Abd-Elhameed, "New algorithm for the numerical solutions of nonlinear third-order differential equations using Jacobi-Gauss collocation method," Mathematical Problems in Engineering, vol. 2011, Article ID 837218, 14 pages, 2011.

[16] A. H. Bhrawy and A. S. Alofi, "A Jacobi-Gauss collocation method for solving nonlinear Lane-Emden type equations," Communications in Nonlinear Science and Numerical Simulation, vol. 17, no. 1, pp. 62-70, 2012.

[17] E. H. Doha and A. H. Bhrawy, "Efficient spectral-Galerkin algorithms for direct solution of fourthorder differential equations using Jacobi polynomials," Applied Numerical Mathematics., vol. 58, no. 8, pp. 1224-1244, 2008.

[18] E. H. Doha, A. H. Bhrawy, and R. M. Hafez, "A Jacobi-Jacobi dual-Petrov-Galerkin method for thirdand fifth-order differential equations," Mathematical and Computer Modelling, vol. 53, no. 9-10, pp. 1820-1832, 2011.

[19] E. H. Doha, A. H. Bhrawy, and R. M. Hafez, "A Jacobi dual-Petrov-Galerkin method for solving some odd-order ordinary differential equations," Abstract and Applied Analysis, vol. 2011, Article ID 947230, 21 pages, 2011.

[20] E. H. Doha, "On the coefficients of differentiated expansions and derivatives of Jacobi polynomials," Journal of Physics A, vol. 35, no. 15, pp. 3467-3478, 2002.

[21] E. H. Doha, "On the construction of recurrence relations for the expansion and connection coefficients in series of Jacobi polynomials," Journal of Physics A, vol. 37, no. 3, pp. 657-675, 2004.

[22] Y. Luke, The Special Functions and Their Approximations, vol. 2, Academic Press, New York, NY, USA, 1969.

[23] I. H. Abdel-Halim Hassan, "Differential transformation technique for solving higher-order initial value problems," Applied Mathematics and Computation, vol. 154, no. 2, pp. 299-311, 2004.

[24] D. K. Salkuyeh, "Convergence of the variational iteration method for solving linear systems of ODEs with constant coefficients," Computers E Mathematics with Applications, vol. 56, no. 8, pp. 2027-2033, 2008. 


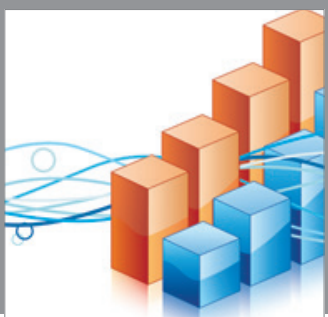

Advances in

Operations Research

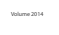

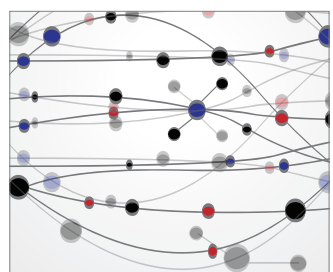

\section{The Scientific} World Journal
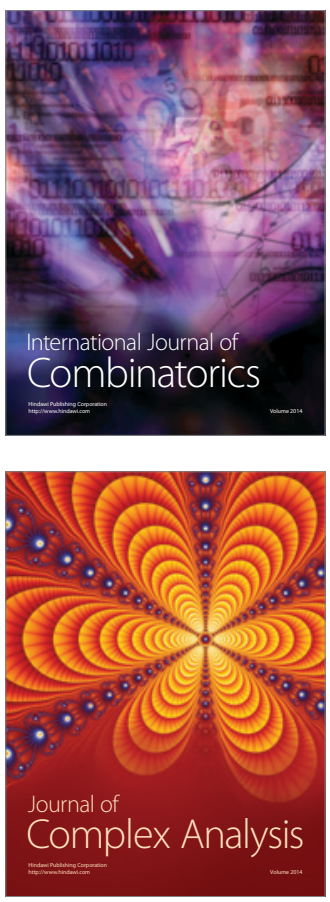

International Journal of

Mathematics and

Mathematical

Sciences
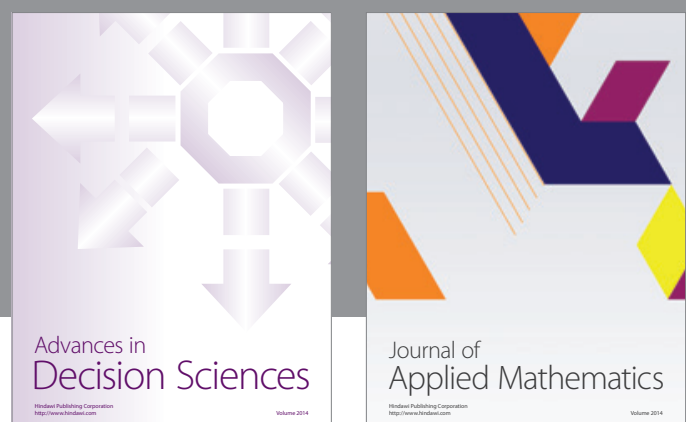

Journal of

Applied Mathematics
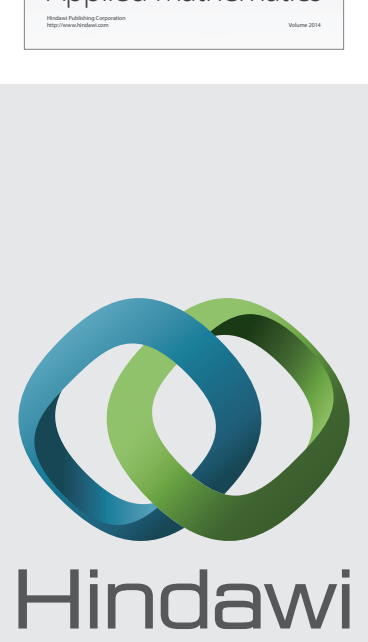

Submit your manuscripts at http://www.hindawi.com
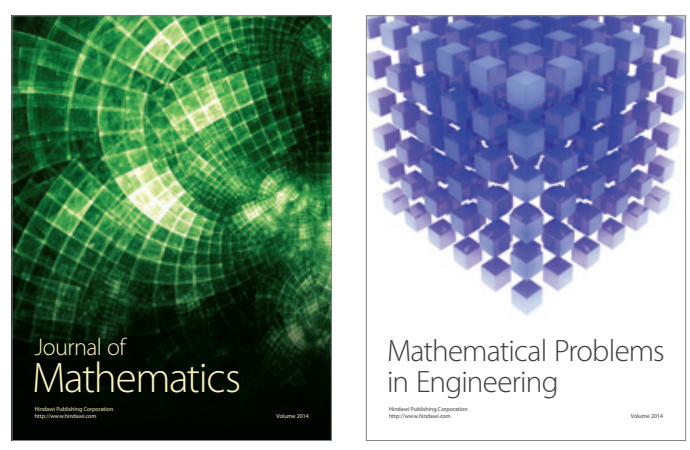

Mathematical Problems in Engineering
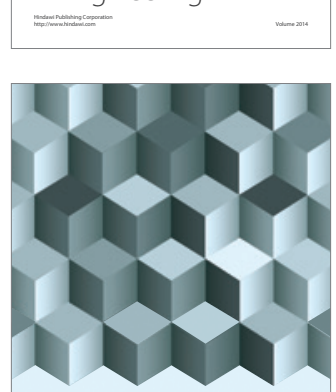

Journal of

Function Spaces
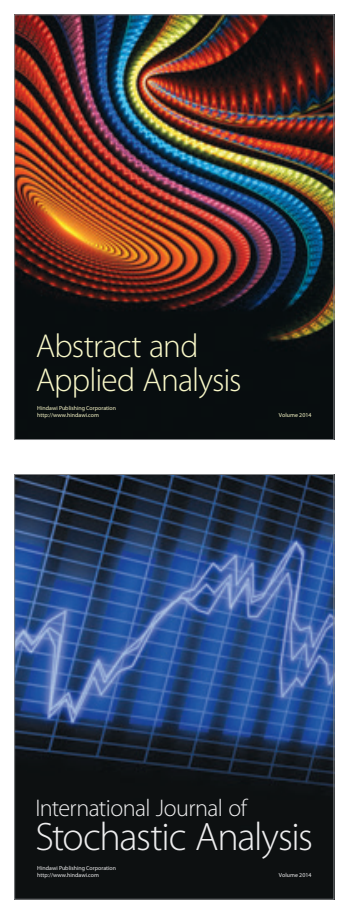

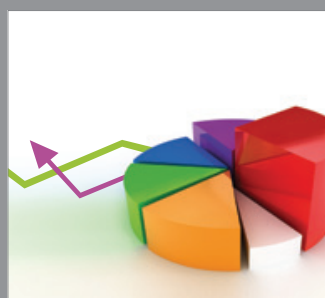

ournal of

Probability and Statistics

Promensencen
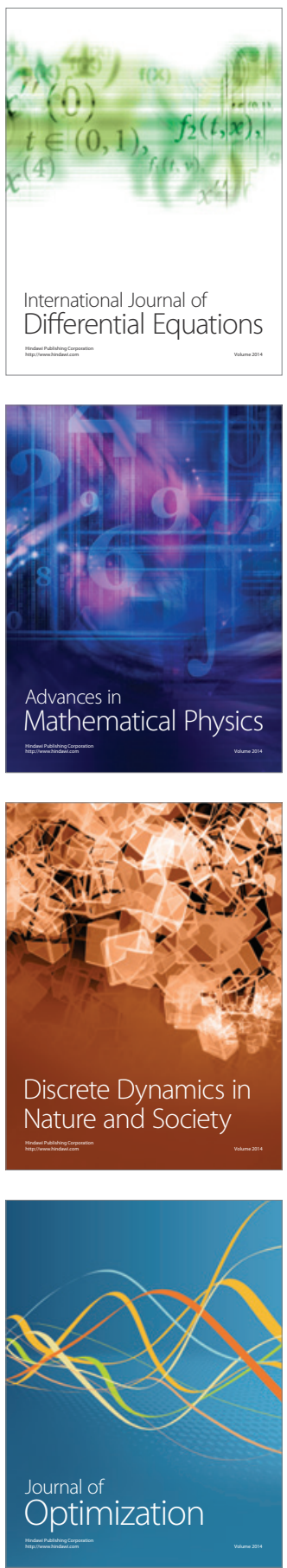